\title{
Placenta accreta syndrome: A rising epidemic in obstetrics
}

\author{
Savita S Patil, Shaila S Puranik, Sharvari D Vishwasrao \\ Correspondence: Dr Savita S Patil, Lecturer, Department of Pathology, B J Govt Medical \\ College and Sassoon General Hospital, Pune. India; Email - drsavitapatil@gmail.com
}

Distributed under Creative Commons Attribution-Share Alike 4.0 International.

\begin{abstract}
Objectives: The aims of our study were to determine incidence of placenta accreta syndrome and correlate it with the risk factors. Methods: A clinicohistopathological study had been done on the basis of clinical, radiological and histopathogical analysis. Obstetric hysterectomy specimens were studied and those diagnosed as placenta accreta syndrome were investigated in detail for history of Caesarean section. Results: The incidence of placenta accreta syndrome is 4.3 per 10,000 deliveries. The study revealed the placenta accreta syndrome in younger age group (mean age $27 \mathrm{yrs}$ ) and showed its relation with risk factors such as previous caesarean section ( $76 \%$ cases), placenta praevia (53\% cases) and multiparity (92\%). Conclusions: With high index of suspicion in presence of known risk factors and appropriate radiological investigations, this syndrome can be diagnosed antenatally and planned efforts to avoid life threatening haemorrhage and early hysterectomy can be made.
\end{abstract}

Keywords: Placenta accreta, caesarean section, placenta praevia, multiparity.

Placenta accreta syndromes have evolved into one of the most serious problems in obstetrics. Placenta accreta refers to a condition in which the placental villi adhere to underlying myometrium due to partial or complete absence of nitabuchs layer ${ }^{1}$. Decidual formation is defective over a previous hysterotomy scar or instrumentation sites such as curettage. Previous Caesarean section, concurrent placenta praevia and uterine surgery are some of the risk factors. Placenta accreta refers to an adherent placenta while placenta increta refers to invasion of the myometrium by placental villi and placenta percreta refers to invasion of the villi upto the serosal layer. This syndrome contributes significantly to maternal morbidity and mortality. Also accreta syndromes are one of the leading causes of intractable post partum haemorrhage and emergency peripartum hysterectomy. Recent studies have demonstrated increased incidence of placenta accreta syndrome $^{2,3}$. Anterpartum diagnosis can be made using colour Doppler as early as second trimester. Diagnostic features include absence of normal hypoechoic boundary between placenta and serosa or urinary bladder ${ }^{4}$. The aims of this study were to determine the incidence of accreta syndrome and to observe the risk factors of it.

\section{Materials and Methods}

This hospital based retrospective study was carried out from 2013 to 2016 on specimens received in surgical pathology section. The specimens were fixed in formalin, trimmed and stained with routine haematoxylin and eosin stain. Clinical and pathological correlation was done by obtaining relevant clinical details, radiological findings and histopathological diagnosis. Appropriate permissions

Received: 22 nd September 2017. Accepted: 30 th November 2017.

Patil SS, Puranik SS, Vishwasrao SD. Placenta accreta syndrome: A rising epidemic in obstetrics. The New Indian

Journal of OBGYN. 2018; 4(2): $138-40$. 
were taken from the institutional ethical committee.

\section{Results}

Number of specimens received was 13 . The incidence is 4.3 per 10,000 deliveries. Age range of patients was 22 -

\begin{tabular}{ll} 
Table 1: Different risk factors (N=13) \\
\hline Risk factors & Number (\%) \\
\hline Previous LSCS & $10(76 \%)$ \\
Previous uterine surgery & $1(13 \%)$ \\
Concurrent placenta praevia & $7(53 \%)$ \\
Multiparity & $12(92 \%)$ \\
Age $>35$ years & $1(7 \%)$ \\
\hline
\end{tabular}

38 years, mean age being 27 years. History of previous caesarean section was present in 10 cases. History of

\section{Discussion}

Placenta Accreta has an incidence of 1 in 2500 deliveries as per Morgan $\mathrm{M}$ et al ${ }^{5}$ and 1.7 per 10,000 deliveries as per Fitzpatrick et al ${ }^{3}$. Incidence in this study is 4.3 per 10,000 deliveries. This high incidence could be attributable to our centre being tertiary referral centre for a population of over 10 million. In spite of considering this factor, higher incidence of placenta accreta syndrome in recent years has prompted us to document our case series.

The increased frequency of placenta accreta has been associated with liberalized use of caesarean section. Other significant risk factors include multiparity, placenta praevia, advanced age, Asherman syndrome, submucous myoma, previous curettage and chronic hypertension ${ }^{4}$.

Out of these, caesarean section and placenta praevia are the major risk factors. In this study, majority of the cases were from second decade with the

mean age of 27 concurrent placenta praevia was present in 7 cases. History of previous uterine surgery other than caesarean section was given in 1 case. Twelve patients out of 13 were multiparous (Table 1).

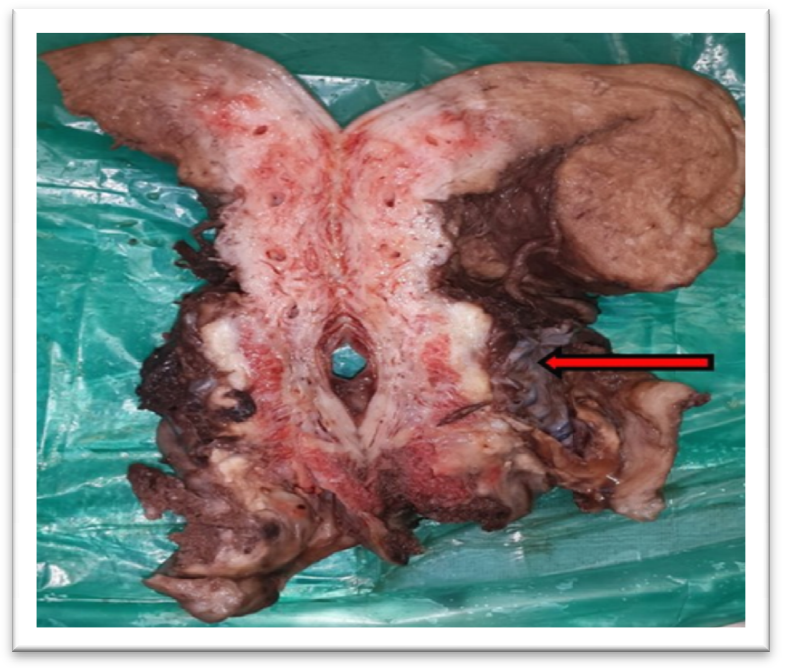

Figure 1: Placenta Accreta
$113(84 \%)$

$39(29 \%)$

$86(64 \%)$

$77(55 \%)$ years. In contrast to the study done by Fitzpatrick et al ${ }^{3}$, our patients were from the younger age group, this being an alarming sign as younger patient have to face morbidity of emergency caesarean section, following dreadful postpartum haemorrhage. Also the social fallout of an early hysterectomy can be considerable.

Most of patients were multiparous with three being $G_{4}$, five being $G_{3}$, three being $G_{2}$ and one being $G_{1}$. Thus 12 out of 13 were multiparous (92\%) and similar findings were recorded in the study by Choudhary $\mathrm{D}$ et $\mathrm{al}^{2}$ where all patients were multiparous (Table 2). Present study showed history of previous caesarean section in $76 \%$ cases, which highlights the established risk associated with it, in comparison it is $85 \%$ in study by Chaudhary D et $\mathrm{al}^{2}$ and $84 \%$ in study by Fitzpatrick KE et $\mathrm{al}^{3}$ (Table 2). Also concurrent placenta praevia was recorded in 53\% cases, which is lesser compared to $71 \%$ in study by Choudhary et al ${ }^{2}$ and $64 \%$ in study by Fizpatrick KE et al ${ }^{3}$ (Table 2). 


\section{Conclusion}

The increased frequency of placenta accreta has been associated with liberalized use of caesarean section. Antepartum diagnosis by appropriate radiological investigations can be made and patient can be saved from life threatening postpartum haemorrhage well as planned efforts to save the uterus can be made.

\section{Conflict of interest: None. Disclaimer: Nil.}

\section{References}

1.Cunningham FG, Leveno KJ, Bloom SL, Spong CY, Dashe JS, Hoffman BL, et al. Williams Obstetrics. 24th edition. New York: McGraw Hill; 2014.

2.Choudhary D, Nigam A, Yadav R, Choudhary S. Placenta Accreta: Obstetricians nightmare. A case series of 7 patients. NJOG. 2012; 7(3): 56-8.
3.Fitzpatrick KE, Sellers S, Spark P, Kurinczuk JJ, Brocklehurst P, Knight M. Incidence and risk factors for Placenta Accreta/ Increta / Percreta in UK: a national case control study. PLoS ONE. 2012; 7(12): e 5283. doi 10.137// journal pone.0052893

4.Comstock $\mathrm{CH}$. Antenatal diagnosis of placenta accreta: a review. Ultrasound Obstet Gynaecol. 2005; 26 (1): 89-96.

5.Morgan M, Atalia R. Mifepristone and misoprostol for the management of placenta accrete - a new alternative approach. BJOG. 2009; 116(7): 1002-3.

\section{Savita S Patil ${ }^{1}$, Shaila S Puranik ${ }^{2}$, Sharvari D Vishwasrao $^{3}$}

${ }^{1}$ Lecturer, Department of Pathology; ${ }^{2}$ Professor and Head, Department of Pathology; ${ }^{3}$ Post Graduate Resident, Department of Pathology, B J Govt Medical College and Sassoon General Hospital, Pune, India. 